\title{
A view from home
}

\begin{abstract}
Andrew M. Sessler, Director of the Lawrence Berkeley Laboratory ( LBL) at the University of California, Berkeley, responds here to the assessment of LBL carried by Nature (August 12, page 528)
\end{abstract}

THE article on LBL was, in my opinion, neither fair nor accurate. I acknowledge the right of a writer to put down what he believes he has observed, but I cannot in good conscience say that I recognise our Laboratory either in its past or present state. In describing its past, for example, the writer, Wil Lepkowski, quotes Robert Yaes on the failures of Lawrence's Laboratory to discover artificial radioactivity and nuclear fission. Those are true charges, but they should surely be considered against a background of the many revolutionary discoveries which were made in the 1930s when Lawrence's Laboratory led the world in nuclear science.

Many fundamental features of nuclear reactions, nuclear structure, radioactive decay, nuclear chemistry and artificial radioactivity were established. The Laboratory was the birthplace of the transuranium elements, and has active research currently in progress to search for possible superheavy elements. The Laboratory established the science of nuclear medicine before the Second World War, when most of the radioactive tracers employed in medicine today were discovered and the foundations were set for the tracer techniques and scintillation detector cameras that are now so commonly used in diagnostic medicine. LBL's eight Nobel Prizes in fact reflect its vitality in basic science throughout Lawrence's time.

Mr Lepkowski is correct in his point that LBL no longer dominates the world of high energy physics, but the sense of failure he attaches to this seems unwarranted. It would be absurd to expect that any laboratory could continue to dominate such a thriving field. Yet our high energy physicists from LBL are not in fact doing all that badly. When the Nature article appeared the high energy physics community throughout the world was excitedly discussing the first observation of a charmed particle by a long-standing Lawrence Berkeley Laboratory-Stanford Linear Accelerator collaborative group. This same collaboration had made a major discovery two years earlier of the $\psi / \mathrm{J}$ particle, at the same time that the same particle was discovered at Brookhaven, and subsequently they discovered the $\psi^{\prime}$. Furthermore, the collaboration of LBL and SLAC is a joint project in every sense of the word: they have been working together on this project for more than five years, using a national facility supported by the entire high energy physics community in the United States. LBL is proud to be an essential part of its design, construction and management, and can expect to continue to have a significant role in high energy physics research for decades to come.

Mr Lepkowski overlooks the strong and unique position that LBL occupies in heavy-ion nuclear physics. Our Laboratory has major programmes at its sector-focusing 88-inch cyclotron, the SuperHILAC and the Bevalac, the latter being the only source of relativistic heavy ions in the world. LBL accelerators are operated as national facilities and there are strong in-house experimental and theoretical groups. We are not blind to the fact that formidable competition is growing, particularly in Europe which is now allocating far more support to nuclear physics than is the United States, but at this time LBL is still considered by all to be a major international centre for nuclear physics.

Basic research is also very healthy at LBL in fields too numerous to catalogue in detail. Fundamental studies in biology and medicine work range from yeast genetics, physiology, and lipoprotein studies to radiobiology and nuclear medicine. LBL, in collaboration with others, is currently pioneering clinical trials on the efficiency of relativistic-energy heavy ions in cancer therapy. In molecular science we have some of the world's forefront studies on reactions in crossed molecular beams, on laser induced chemistry, and on quantum electrodynamic studies in high- $Z$ hydrogenlike atoms.

In material science surface polaritons and plasmons are being studied by means of their nonlinear excitation through optical mixing of laser beams; and synchroton radiation is being employed, along with low energy electron diffraction (LEED), to ascertain the nature of catalytic surfaces. The fundamental understanding of alloys by use of electron microscopy and other advanced techniques has led to the ability to design steels and alloys of unprecedented toughness and hardness. From the field of astronomy there is the first measurment of the mass of a neutron star. In sum, basic science at LBL is diverse, excellent and enthusiastically pursued.

Mr Lepkowski dwells negatively on changes going on in LBL to address changing national and societal needs and a new set of goals from its major funding agency, the US Energy Research and Development Administration. Adaptation to a changing world is a necessity for organisations as it is for individuals. Furthermore, it is simply false to state that the old leaders and, in particular, the Nobelists, are still the dominating leaders of the Laboratory. In fact the Director, the Deputy Director, and eight of the ten Associate Directors have held their positions for less than four years. We of the present management make no apologies for changing the character at LBL.

We can also point to numerous entries into new fields in which important contributions have already been made and which have exciting prospects for the future. Among these are geoscience, geothermal resource assessment, controlled thermonuclear power, metallurgy, catalysis, isotope separation, materials science, environmental and biological effects of energy technology pollutants, energy policy analysis, solar power, and energy conservation studies. In our experience, when we enter fields which are nontraditional for LBL the quality of our scientific staff is revealed by immediate innovative use of advanced experimental techniques and theoretical analysis which result in important or revolutionary contributions to the new fields.

I can give the example of photoelectron spectroscopy turned to the study of the role of power plant particulates in the catalytic conversion of SO, to (harmful) sulphates; or the development, by a scientist previously working on the Lamb Shift, of the most sensitive detector for heavy metals by Isotope Shifted Zeeman Atomic Absorption (IZAA): or the development of superconducting quantum interference devices (SQUID) to a sensitivity some orders of magnitude greater than that available elsewhere, and their direct application to geothermal resource exploration. Or I could cite our work, some years ago, which initiated concern over the catalytic destruction of the protective ozone layer by supersonic transports.

I could give several examples, but let it suffice to say that we feel comfortable in our new activities because we know we are doing excellent science and engineering on problems of importance to mankind. 